\title{
Objective and Violation Upper Bounds on a DIRECT-filter Method for Global Optimization
}

\author{
M. Fernanda P. Costa ${ }^{1[0000-0001-6235-286 X]}$, \\ Ana Maria A. C. Rocha ${ }^{2[0000-0001-8679-2886]}$, and \\ Edite M. G. P. Fernandes 2 [0000-0003-0722-9361] \\ 1 Centre of Mathematics, \\ University of Minho, 4710-057 Braga, Portugal \\ mfc@math.uminho.pt \\ 2 ALGORITMI Center, \\ University of Minho, 4710-057 Braga, Portugal, \\ \{arocha, emgpf\}@dps.uminho.pt
}

\begin{abstract}
This paper addresses the problem of solving a constrained global optimization problem using a modification of the DIRECT method that incorporates the filter methodology to simultaneously minimize the objective function and the constraints violation. Thus, in the "Selection" step of the herein proposed DIRECT-filter algorithm, the hyperrectangles are classified in four categories and subsequently handled separately. The new algorithm also imposes upper bounds on the objective function and constraints violation aiming to discard some hyperrectangles from the process of identifying the potentially optimal ones. A heuristic to avoid the exploration of the hyperrectangles that have been mostly divided is also implemented. Preliminary numerical experiments are carried out to show the effectiveness of the imposed upper bounds on the objective and violation as well as the goodness of the heuristic.
\end{abstract}

Keywords: Global Optimization · DIRECT · Filter Method · Heuristic

\section{Introduction}

The paper aims to address the use of the filter methodology [1] combined with a DIRECT-type method [2] to globally solve non-smooth and non-convex constrained optimization problems. The constrained global optimization (CGO) problem has the form:

$$
\begin{aligned}
\min _{x \in \Omega} & f(x) \\
\text { subject to } & h(x)=0 \\
g(x) & \leq 0,
\end{aligned}
$$

where $f: \mathbb{R}^{n} \rightarrow \mathbb{R}, h: \mathbb{R}^{n} \rightarrow \mathbb{R}^{m}$ and $g: \mathbb{R}^{n} \rightarrow \mathbb{R}^{p}$ are nonlinear continuous functions and $\Omega=\left\{x \in \mathbb{R}^{n}:-\infty<l_{i} \leq x_{i} \leq u_{i}<\infty, i=1, \ldots, n\right\}$. Since convexity is not assumed, many local minima may exist in the feasible region, although we require only a global solution. For non-smooth problems, 
the derivative-free methods are the most appropriate. Popular methods to solve problem (1) combine a penalty term, which depends on a constraint violation measure, with the objective function to give the so-called penalty function. The penalty term aims to penalize $f$ whenever an approximation point is found that is infeasible. Penalty functions within a DIRECT-type framework are proposed in $[3,4]$. An auxiliary function that combines in a special manner information on the objective and constraints is presented in [5]. Other techniques that involve the handling of the objective function and constraints violation separately can be found in $[6,7,8]$.

This paper addresses the exploration of the DIRECT method in order to solve CGO problems. It uses the filter methodology [1] to handle the constraints. The objective function and the constraints violation measure are separately handled and both simultaneously minimized. The main differences relative to the work reported in [8] are the following:

1. four categories of hyperrectangles (according to the violation measure and the non-dominance $v s$ dominance feature of their center points) are defined instead of three;

2. upper bounds on the objective and violation values are imposed during the selection step in order to reduce the number of explored hyperrectangles;

3. a heuristic is used to prevent the mostly divided hyperrectangles to be selected and identified as potentially optimal.

The paper is organized as follows. Section 2 briefly presents some ideas and the main steps of the DIRECT method. Section 3 describes the proposed extension to handle CGO problems, in particular, the use of a filter method to classify each hyperrectangle according to its non-dominance/dominance feature and constraints violation magnitude. Further, the strategy that imposes upper bounds on $f$ and violation values, as well as the heuristic are exposed. Finally, Sect. 4 contains the results of our preliminary numerical experiments and we conclude the paper with the Sect. 5 .

\section{Features about DIRECT Method}

The DIRECT (DIviding RECTangles) algorithm, originally proposed to solve bound constrained global optimization problems, assumes that the objective function, $f$, is a continuous function and creates finer and finer partitions of the hyperrectangles generated from the set $\Omega[2,9,10]$. The algorithm is a modification of the standard Lipschitzian approach, in which $f$ is assumed to satisfy the Lipschitz condition

$$
\left|f\left(x_{1}\right)-f\left(x_{2}\right)\right| \leq K\left\|x_{1}-x_{2}\right\| \text { for all } x_{1}, x_{2} \in \Omega,
$$

where the Lipschitz constant $K>0$ is viewed as a weighting parameter that indicates how much emphasis to place on global versus local search. 
DIRECT is a deterministic and derivative-free method that is able to explore optimal regions aiming to converge to the global optimum and at the same time avoiding being trapped in a local optimum.

DIRECT is described by six main steps: "Initialization", "Selection", "Sampling", "Division", "Iteration" and "Termination" $[2,11,12]$.

The "Selection" step serves the purpose of identifying the set of indices of hyperrectangles that are the most promising, denoted by potentially optimal hyperrectangles (POH), based on the current partition of $\Omega$. In the "Sampling" steps, the set of dimensions with the maximum size in each $\mathrm{POH}$ is identified to define points where the objective function is evaluated. For the "Division" step, DIRECT uses two measures: (i) the size of the hyperrectangle to favor the global search feature of the algorithm; (ii) the value of the hyperrectangle to give preference to the local search feature. The value corresponds to the objective function value alone at the center, for bound constrained problems (and to the objective function and constraint violation values, when problem (1) is addressed).

For further details on the original DIRECT and other recent interesting modifications, we refer the reader to $[13,14,15,16,17,18,19,20]$.

\section{DIRECT-filter Method}

In this section, we reveal how the DIRECT algorithm is extended to incorporate the filter methodology in order to minimize both the objective function and constraints violation. First, we briefly present the filter methodology and the proposed extensions to be incorporated in the main steps of DIRECT. Second, the strategy that uses the upper bounds on objective and violation values and the heuristic to avoid the selection of the mostly divided hyperrectangles are presented.

\subsection{Filter Methodology}

Based on the filter methodology [1,21], the problem (1) is reformulated into the following bound constrained bi-objective optimization problem:

$$
\min _{x \in \Omega}(\theta(x), f(x)),
$$

where $\theta(x)=\|h(x)\|_{1}+\left\|g(x)_{+}\right\|_{1}$ is a non-negative function to measure equality and inequality constraints violation, and $g_{+} \in \mathbb{R}^{p}$ is defined componentwise by $\max \left\{0, g_{i}\right\}, i=1, \ldots, p$. A point $x$ is feasible when $\theta(x)=0$ and is infeasible when $\theta(x)>0$. While minimizing the constraints violation, $\theta$, and the objective function, $f$, the filter method builds a region of dominated points that will not be accepted as new approximations to the solution. The concept of dominance arises from the multi-objective optimization area: 
Definition 1. A point $x$, or the corresponding pair $(\theta(x), f(x))$, is said to dominate $y$, or the corresponding pair $(\theta(y), f(y))$, denoted by $x \prec y$, if and only if

$$
\theta(x) \leq \theta(y) \text { and } f(x) \leq f(y)
$$

with at least one inequality being strict.

The filter $\mathcal{F}$ contains a finite set of pairs $(\theta(x), f(x))$, none of which is dominated by any of the others, and the corresponding points $x$ are known as the nondominated points [1].

Let $x^{(k)}$ be a trial point (approximation to the optimal solution of the CGO problem (1)) and $\mathcal{F}_{k}$ be the filter, at iteration $k$, of the algorithm. To avoid the acceptance of the trial point, or the corresponding pair $\left(\theta\left(x^{(k)}\right), f\left(x^{(k)}\right)\right)$, that is arbitrary close to the boundary of the filter, the conditions of acceptability define an envelope around the filter and are as follows:

$$
\theta\left(x^{(k)}\right) \leq(1-\gamma) \theta\left(x^{l}\right) \text { or } f\left(x^{(k)}\right) \leq f\left(x^{l}\right)-\gamma \theta\left(x^{l}\right)
$$

for all points $x^{l}$ that correspond to pairs $\left(\theta\left(x^{l}\right), f\left(x^{l}\right)\right)$ in the filter $\mathcal{F}_{k}$, where $\gamma \in(0,1)$ is fixed. When the point is acceptable to the filter, the filter is updated and whenever a point is added to the filter, all the dominated points are removed from the filter.

We note that the filter contains only infeasible points. However, the feasible point with the least function value, denoted by $f_{\text {best }}$, is saved and is used to filter other feasible points.

\subsection{Identifying $\mathrm{POH}$ in the DIRECT-filter Method}

In the context of solving a CGO problem, the herein proposed algorithm defines two separate regions within the usually called infeasible region. One is denoted by "infeasible" region (identified by $I$ ) and contains hyperrectangles with center points $c_{j}$ that satisfy $\theta\left(c_{j}\right)>\theta_{\text {feas }}$, for a sufficiently small positive tolerance $\theta_{\text {feas }}$. The other is called "feasible-band" region (identified by $F B$ ) and contains the hyperrectangles with center points that satisfy $0<\theta\left(c_{j}\right) \leq \theta_{\text {feas }}$. On the other hand, the herein coined "feasible" region (identified with $F$ ) contains hyperrectangles with $\theta\left(c_{j}\right)=0$.

When applying a DIRECT-type method, in the partition of $\left\{H^{i}: i \in I_{k}\right\}$ of iteration $k$, using the filter methodology and the three regions above defined, the identification of $\mathrm{POH}$ (in the "Selection" step) is implemented separately for the following four sets of indices:

- the set $I_{k}^{F B / N D+\mathrm{b}}$, contains indices of hyperrectangles with center points in the "feasible-band" region that are non-dominated $(F B / N D)$, appended with the index of the hyperrectangle that corresponds to $f_{\text {best }}(+\mathrm{b})$;

- the set $I_{k}^{F B / D+F \backslash \mathrm{b}}$ contains the indices of hyperrectangles with center points in the "feasible-band" region that are dominated $(F B / D)$, appended with the indices of the hyperrectangles with centers in the region $F$ except $\mathrm{b}$ $(+F \backslash \mathrm{b})$; 
- the set $I_{k}^{I / N D}$ contains the indices of hyperrectangles with non-dominated center points that are in the "infeasible" region $(I / N D)$;

- the set $I_{k}^{I / D}$ contains the indices of hyperrectangles with dominated center points that belong to the "infeasible" region $(I / D)$.

As usual, the hyperrectangles are organized by groups of the same size. The proposed strategy aims to identify, from each hull, indices of promising hyperrectangles, in terms of the

- optimality measure $f$, when the indices for exploration belong to the sets $I^{F B / N D+\mathrm{b}}$ and $I^{F B / D+F \backslash \mathrm{b}}$;

- feasibility measure $\theta$, when the indices belong to the sets $I^{I / N D}$ and $I^{I / D}$.

Thus, in this filter-type method context, the algorithm identifies $\mathrm{POH}$ with respect to (w.r.t.) $f$, using the following definition [2]:

Definition 2. Given the partition $\left\{H^{i}: i \in I\right\}$ of $\Omega$, let $\epsilon$ be a positive constant and let $f_{\min }$ be the current best function value among center points in the regions "feasible" and "feasible-band". A hyperrectangle $j$ is said to be potentially optimal w.r.t. $f$ if there exists some rate-of-change constant $\hat{K}>0$ such that

$$
\begin{aligned}
& f\left(c_{j}\right)-\hat{K} d_{j} \leq f\left(c_{i}\right)-\hat{K} d_{i}, \text { for all } i \in I \\
& f\left(c_{j}\right)-\hat{K} d_{j} \leq f_{\min }-\epsilon\left|f_{\min }\right|
\end{aligned}
$$

where $c_{j}$ is the center, $d_{j}$ is a measure of the size of the hyperrectangle $j$ (for instance, the distance from $c_{j}$ to its vertices) and $I$ is $I^{F B / N D+b}$ or $I^{F B / D+F \backslash b}$.

The value of $f_{\min }$ coincides with $f_{\text {best }}$ if there are center points with $\theta=0$; otherwise $f_{\min }$ is set to the least function value of the center points in the region $F B / N D$.

On the other hand, for the remaining sets of indices (hyperrectangles) where $\theta$ is used to define the hull, the algorithm identifies $\mathrm{POH}$ w.r.t $\theta$, by adopting the following definition $[6,8]$ :

Definition 3. Given the partition $\left\{H^{i}: i \in I\right\}$ of $\Omega$, let $\epsilon$ be a positive constant. A hyperrectangle $j$ is said to be potentially optimal w.r.t. the function $\theta$ if there exists some constant $\hat{K}>0$ such that

$$
\begin{aligned}
& \theta\left(c_{j}\right)-\hat{K} d_{j} \leq \theta\left(c_{i}\right)-\hat{K} d_{i}, \text { for all } i \in I \\
& \theta\left(c_{j}\right)-\hat{K} d_{j} \leq \theta_{\min }-\epsilon \theta_{\min }
\end{aligned}
$$

where $\theta_{\min }>0$ is the $\theta$ value that corresponds to $f_{\min }$ if the "feasible-band" region is non-empty; otherwise is the least value of $\theta$ reached by a point in the "infeasible" region. The set $I$ is $I^{I / N D}$ or $I^{I / D}$. 


\subsection{Objective and Violation Upper Bounds}

We now show how upper bounds on objective function and constraints violation, denoted by $f^{U}$ and $\theta^{U}$ respectively, are imposed in a way that hyperrectangles with $f$ and/or $\theta$ values greater than the corresponding upper bounds are not considered in the "Selection" step to identify POH. The bounds $f^{U}$ and $\theta^{U}$ are defined at each iteration and depend on the information available at that moment.

Thus, the bound on $f$ to apply to the set $I^{F B / D+F \backslash \mathrm{b}}$ is defined by

$$
f_{F B}^{U}=f_{F B}+\beta_{f}\left|f_{F B}\right| \text { with } f_{F B}=\max \left\{f_{\max }^{F B / N D}, f_{\text {best }}\right\},
$$

where $f_{\max }^{F B / N D}$ - directly identified from the filter $\mathcal{F}$ - is the $f$ value of the center of the hyperrectangle with the lowest $\theta$ value among the hyperrectangles with center in the region $F B / N D$, and $\beta_{f} \geq 0$ is a constant factor.

On the other hand, the bound on $f$ to apply to the set $I^{I / D}$ is defined by

$$
f_{I}^{U}=f_{I}+\beta_{f}\left|f_{I}\right|
$$

where $f_{I}$ - directly identified from the filter - is the $f$ value of the center of the hyperrectangle with the lowest $\theta$ value among the hyperrectangles with center in the region $I / N D$. This $\theta$ value will be denoted by $\theta_{\min }>\theta_{\text {feas }}$.

Moreover, $\theta^{U}$ is computed using $\theta_{\min }$ as follows:

$$
\theta^{U}=\theta_{\min }+\beta_{\theta} \theta_{\min }
$$

where $\beta_{\theta}>0$ is a constant factor. This upper bound on $\theta$ is applied only to the sets $I^{I / N D}$ and $I^{I / D}$, since the other two are naturally bounded by $\theta_{\text {feas }}$.

From hereafter, we denote the basic DIRECT-filter method (as described in the previous subsection) by "DIRECT-f" and the variant that incorporates the upper bounds on $f$ and $\theta$ (as reported here in this subsection) by "UB-DIRECTf".

\subsection{Heuristic}

Besides using the above described upper bounds, the "UB-DIRECT-f" algorithm can be enhanced with a heuristic that aims to avoid identifying $\mathrm{POH}$ among those hyperrectangles that were mostly divided [17].

The heuristic is applied only to the two sets of indices $I^{F B / D+F \backslash \mathrm{b}}$ and $I^{I / D}$. Thus, hyperrectangles with indices based on size that are larger than $\left\lfloor i_{b} / 4\right\rfloor$ are discarded, where $\lfloor t\rfloor$ gives the greatest integer less than or equal to $t$, and $i_{b}$ is the index based on the size of the hyperrectangle that corresponds to

- $f_{\text {min }}$, when the hull from the set $I^{F B / D+F \backslash \mathrm{b}}$ is explored;

$-\theta_{\text {min }}$, when the hull from the set $I^{I / D}$ is explored. 
(We note that the larger the size, the smaller is the index based on size.)

This heuristic runs for a cycle of 10 iterations and aims to potentiate the exploration of hyperrectangles of larger sizes in order to identify $\mathrm{POH}$. With this selection, global information during the search is reinforced and the likelihood is that $f_{\min }$ and/or $\theta_{\min }$ may be improved. This cycle of iterations is implemented every 10 iterations of the original "UB-DIRECT- $f$ ". While the heuristic is active, the upper bounds on $f$ and $\theta$ are disabled. This variant is denoted by "UBDIRECT-f+Heur" in the subsequent tables of results.

\section{Numerical Experiments}

During the preliminary numerical experiments, a set of benchmark problems is used. The MATLAB ${ }^{\circledR}$ (MATLAB is a registered trademark of the MathWorks, Inc.) programming language is used to code the algorithm and the tested problems.

Unless otherwise stated, the stopping conditions for the algorithm are the following. We consider that a good approximate solution $x^{(k)}$, at iteration $k$, is found, if the conditions

$$
\theta\left(x^{(k)}\right) \leq \eta_{1} \text { and } p_{\text {error }} \equiv \frac{\left|f\left(x^{(k)}\right)-f^{*}\right|}{\max \left\{1,\left|f^{*}\right|\right\}} \leq \eta_{2}
$$

are satisfied, for sufficiently small tolerances $\eta_{1}, \eta_{2}>0$, where $f^{*}$ is the best known solution to the problem. However, if conditions (6) are not satisfied, the algorithm runs until a maximum number of function evaluations, $n f e_{\max }$, is reached.

The parameter values for the algorithm are set as follows: $\gamma=1 E-05$, $\theta_{\text {feas }}=1 E-04, \epsilon=1 E-04, \beta_{f}=1.1, \beta_{\theta}=1 E+04, \eta_{1}=1 E-04, \eta_{2}=1 E-04$ and $n f e_{\max }=1 E+06$. (We note that a smaller value of $\beta_{f}$ was also tested but the reported choice gave better results specially for the larger problems.)

Our goal is to reveal the effectiveness of the proposed objective function and constraint violation upper bounds in reducing the computational burden without affecting the robustness of the DIRECT-filter method.

Table 1 presents a comparison of our solutions with others reported in the literature, when solving the problem "Gomez \#3" [5], with global optimum value $f^{*}=-0.9711$, occurring at $(0.109,-0.623)$ :

$$
\begin{aligned}
& \min _{x \in \Omega}\left(4-2.1 x_{1}^{2}+\frac{x_{1}^{4}}{3}\right) x_{1}^{2}+x_{1} x_{2}+\left(-4+4 x_{2}^{2}\right) x_{2}^{2} \\
& \text { subject to }-\sin \left(4 \pi x_{1}\right)+2 \sin ^{2}\left(2 \pi x_{2}\right) \leq 0
\end{aligned}
$$

with $\Omega=\left\{x \in \mathbb{R}^{2}:-1 \leq x_{i} \leq 1, i=1,2\right\}$. The solutions reported in the table have $1 \%$ and $0.01 \%$ error relative to the known global solution. The results are compared to those available in [5] and to another filter-based DIRECT algorithm (in [8]). We can see that the implementation of the upper bounds on $f$ and $\theta$ as well as the heuristic make the DIRECT-filter method more efficient. 
Table 1. Comparison results when solving problem "Gomez \#3".

\begin{tabular}{rrrrrrr}
\hline algorithm & $p_{\text {error }}$ & $f\left(x^{(k)}\right)$ & $\theta\left(x^{(k)}\right)$ & $k$ & $n f e$ & $f^{*}$ \\
\hline "DIRECT-f" & $1 \%$ & -0.961782 & $0.00 \mathrm{E}+00$ & 9 & 185 & -0.9711 \\
"UB-DIRECT-f" & & -0.961782 & $0.00 \mathrm{E}+00$ & 9 & 225 & \\
"UB-DIRECT-f+Heur" & & -0.961782 & $0.00 \mathrm{E}+00$ & 10 & 149 & \\
in [8] & & - & - & 9 & 219 & \\
in [5] & & - & - & - & 89 & \\
\hline "DIRECT-f" & $0.01 \%$ & -0.971006 & $6.00 \mathrm{E}-05$ & 17 & 615 \\
"UB-DIRECT-f" & & -0.971006 & $6.00 \mathrm{E}-05$ & 17 & 683 & \\
"UB-DIRECT-f+Heur" & & -0.971041 & $3.17 \mathrm{E}-05$ & 18 & 555 \\
in [8] & & - & -18 & 733 & \\
in [5] & & - & - & -513 &
\end{tabular}

To compare the results to those in [4] (variants DIRECT-GLc and DIRECTGLce), problem "T1" (with several instances depending on $n$ ) is used:

$$
\begin{gathered}
\min _{x \in \Omega} \sum_{i=1}^{n} x_{i} \\
\text { subject to } \sum_{i=1}^{n} x_{i}^{2} \leq 6
\end{gathered}
$$

with $\Omega=\left\{x \in \mathbb{R}^{n}:-1 \leq x_{i} \leq 1, i=1, \ldots, n\right\}$. The algorithms stop with the condition $p_{\text {error }} \leq 1 E-04$ alone (or a maximum of $1 E+06$ function evaluations). See Table 2. Although we are not yet able to achieve convergence before $1 E+06$ function evaluations on the larger instances, $n=5$ and $n=6$ of the problem "T1", the results obtained by "UB-DIRECT-f+Heur" for the other instances outperform the others in comparison.

To analyze the quality of the obtained solutions we use problem "5" (available in $[22])$ :

$$
\begin{aligned}
\min _{x \in \Omega} & x_{3} \\
\text { subject to } & 30 x_{1}-6 x_{1}^{2}-x_{3}=-250 \\
& 20 x_{2}-12 x_{2}^{2}-x_{3}=-300 \\
& 0.5\left(x_{1}+x_{2}\right)^{2}-x_{3}=-150
\end{aligned}
$$

with $\Omega=\left\{x \in \mathbb{R}^{3}: 0 \leq x_{1} \leq 9.422,0 \leq x_{2} \leq 5.903,0 \leq x_{3} \leq 267.42\right\}$ and problem " 8 " [22]:

$$
\begin{array}{cl}
\min _{x \in \Omega} & x_{1}^{4}-14 x_{1}^{2}+24 x_{1}-x_{2}^{2} \\
\text { subject to } & x_{2}-x_{1}^{2}-2 x_{1} \leq-2 \\
& -x_{1}+x_{2} \leq 8
\end{array}
$$

with $\Omega=\left\{x \in \mathbb{R}^{2}:-8 \leq x_{1} \leq 10,0 \leq x_{2} \leq 10\right\}$ and stop the algorithm after $k_{\max }=20$ iterations and then after $k_{\max }=50$ iterations. The results are compared to those obtained previously in [8], and are shown in Table 3. On the other hand, to analyze the gain in efficiency of the present algorithm variants, Table 4 reports the best $f$ and $\theta$ values obtained by the algorithms when the stopping conditions in (6) are used. The gain in quality and efficiency of the 
Objective and Violation Upper Bounds on a DIRECT-filter Method

Table 2. Comparison results when solving problem "T1".

\begin{tabular}{|c|c|c|c|c|c|c|}
\hline & algorithm & $f\left(x^{(k)}\right)$ & $\theta\left(x^{(k)}\right)$ & $k$ & $n f e$ & $f^{*}$ \\
\hline \multirow[t]{5}{*}{$n=2$} & "DIRECT-f" & -3.464106 & $9.29 \mathrm{E}-05$ & 14 & 1395 & -3.4641 \\
\hline & "UB-DIRECT-f" & -3.464106 & $9.29 \mathrm{E}-05$ & 14 & 893 & \\
\hline & "UB-DIRECT-f+Heur" & -3.464106 & $5.72 \mathrm{E}-05$ & 13 & 335 & \\
\hline & DIRECT-GLc & - & - & - & 1373 & \\
\hline & DIRECT-GLce & - & - & - & 2933 & \\
\hline \multirow[t]{5}{*}{$n=3$} & "DIRECT-f" & -4.242443 & $0.00 \mathrm{E}+00$ & 28 & 16885 & -4.2426 \\
\hline & "UB-DIRECT-f" & -4.242443 & $0.00 \mathrm{E}+00$ & 35 & 37977 & \\
\hline & "UB-DIRECT-f+Heur" & -4.242443 & $9.17 \mathrm{E}-05$ & 29 & 3233 & \\
\hline & DIRECT-GLc & - & - & - & 26643 & \\
\hline & DIRECT-GLce & - & - & - & 8297 & \\
\hline \multirow[t]{5}{*}{$n=4$} & "DIRECT-f" & -4.898847 & $0.00 \mathrm{E}+00$ & 42 & 151753 & -4.899 \\
\hline & "UB-DIRECT-f" & -4.898847 & $3.42 \mathrm{E}-05$ & 39 & 78859 & \\
\hline & "UB-DIRECT-f+Heur" & -4.898440 & $3.30 \mathrm{E}-05$ & 51 & 36219 & \\
\hline & DIRECT-GLc & - & - & - & 192951 & \\
\hline & DIRECT-GLce & - & - & - & 47431 & \\
\hline \multirow[t]{5}{*}{$n=5$} & "DIRECT-f" & $(-5.470982)$ & $(6.65 \mathrm{E}-05)$ & $(61)$ & $>1 \mathrm{E}+06$ & -5.4772 \\
\hline & "UB-DIRECT-f" & $(-5.470711)$ & $(0.00 \mathrm{E}+00)$ & (63) & $>1 \mathrm{E}+06$ & \\
\hline & "UB-DIRECT-f+Heur" & $(-5.474293)$ & $(1.00 \mathrm{E}-04)$ & $(117)$ & $>1 \mathrm{E}+06$ & \\
\hline & DIRECT-GLc & - & - & - & 253805 & \\
\hline & DIRECT-GLce & - & - & - & 78257 & \\
\hline \multirow[t]{5}{*}{$n=6$} & "DIRECT-f" & $(-5.991770)$ & $(0.00 \mathrm{E}+00)$ & $(45)$ & $>1 \mathrm{E}+06$ & -6.0000 \\
\hline & "UB-DIRECT-f" & $(-5.996647)$ & $(0.00 \mathrm{E}+00)$ & $(50)$ & $>1 \mathrm{E}+06$ & \\
\hline & "UB-DIRECT-f+Heur" & $(-5.988112)$ & $(0.00 \mathrm{E}+00)$ & $(79)$ & $>1 \mathrm{E}+06$ & \\
\hline & DIRECT-GLc & - & - & - & 239697 & \\
\hline & DIRECT-GLce & - & - & - & 135843 & \\
\hline
\end{tabular}

In parentheses, the achieved values when the algorithm stops due to $n f e>1 E+06$.

proposed DIRECT-filter method, in particular when the upper bounds on $f$ and $\theta$, and the heuristic are implemented, have been once more demonstrated with the problems " 5 " and "8". The results reported in [8] and those obtained by variants DIRECT-GLc and DIRECT-GLce in [4] are also used in the comparison.

Figures 1(a), 1(b) and 1(c) show the center points generated by the three variants of the DIRECT-filter method when solving the problem " 8 ". Feasible points are marked with ' + ' (blue) and infeasible points with ' $X$ ' (red). It can be seen that the variant "UB-DIRECT-f+Heur" is more effective in reaching the solution. The points cluster around the global solution, being "UB-DIRECT$\mathrm{f}+$ Heur" the one that concentrates the search the most. Figure 1(d) shows the pairs $(\theta, f)$ corresponding to the center points of all the hyperrectangles generated by variant "UB-DIRECT-f+Heur". Dominated points are marked with 'circle' (red) and non-dominated points (or filter points) are marked with 'full circle' (blue). The smaller plot shows an overview of the filter points. 
Table 3. Quality of the results when solving problems " $5 "$ and " 8 ".

\begin{tabular}{rrrrrrr}
\hline Prob. & algorithm & $k=k_{\max }$ & $f\left(x^{(k)}\right)-f^{*}$ & $\theta\left(x^{(k)}\right)$ & $n f e$ & $f^{*}$ \\
\hline "5" & "DIRECT-f" & 20 & $2.512 \mathrm{E}-04$ & $5.92 \mathrm{E}-03$ & 471 & 201.16 \\
& "UB-DIRECT-f" & & $2.512 \mathrm{E}-04$ & $5.92 \mathrm{E}-03$ & 471 & \\
"UB-DIRECT-f+Heur" & & $2.512 \mathrm{E}-04$ & $5.92 \mathrm{E}-03$ & 379 & \\
in [8] & & $2.512 \mathrm{E}-04$ & $5.92 \mathrm{E}-03$ & $(471)$ & \\
& "DIRECT-f" & 50 & $6.819 \mathrm{E}-04$ & $9.44 \mathrm{E}-05$ & 3307 & \\
"UB-DIRECT-f" & & $6.819 \mathrm{E}-04$ & $9.44 \mathrm{E}-05$ & 2653 & \\
"UB-DIRECT-f+Heur" & & $6.819 \mathrm{E}-04$ & $9.44 \mathrm{E}-05$ & 2167 & \\
in [8] & & $6.819 \mathrm{E}-04$ & $9.55 \mathrm{E}-05$ & $(2827)$ & \\
\hline "8" & & & $9.756 \mathrm{E}-04$ & $0.00 \mathrm{E}+00$ & 881 & -118.70 \\
& "DIRECT-f" & 20 & $9.756 \mathrm{E}-04$ & $0.00 \mathrm{E}+00$ & 873 & \\
"UB-DIRECT-f" & & & $7.611 \mathrm{E}-02$ & $5.08 \mathrm{E}-05$ & 587 & \\
"UB-DIRECT-f+Heur" [8] & & $5.372 \mathrm{E}-02$ & $0.00 \mathrm{E}+00$ & $(717)$ & \\
"DIRECT-f" & 50 & $3.724 \mathrm{E}-03$ & $9.85 \mathrm{E}-05$ & 3363 & \\
"UB-DIRECT-f" & & $3.724 \mathrm{E}-03$ & $9.85 \mathrm{E}-05$ & 2715 & \\
"UB-DIRECT-f+Heur" & & $2.993 \mathrm{E}-03$ & $9.82 \mathrm{E}-05$ & 1971 & \\
in [8] & & $3.623 \mathrm{E}-03$ & $9.62 \mathrm{E}-05$ & $(3333)$ & \\
\hline
\end{tabular}

In parentheses, values computed for the comparison, but not reported in [8].

Table 4. Efficiency when solving problems "5" and "8".

\begin{tabular}{|c|c|c|c|c|c|c|}
\hline Prob. & algorithm & $k$ & $f\left(x^{(k)}\right)$ & $\theta\left(x^{(k)}\right)$ & $n f e$ & $f^{*}$ \\
\hline \multirow[t]{6}{*}{$" 5 "$} & "DIRECT-f" & 30 & 201.159343 & $7.83 \mathrm{E}-05$ & 1015 & 201.16 \\
\hline & "UB-DIRECT-f" & 30 & 201.159343 & $7.83 \mathrm{E}-05$ & 883 & \\
\hline & "UB-DIRECT-f+Heur" & 30 & 201.159343 & $7.83 \mathrm{E}-05$ & 769 & \\
\hline & in $[8]$ & 30 & 201.159343 & 7.83E-05 & 1009 & \\
\hline & DIRECT-GLc & - & 201.1593 & - & 819 & \\
\hline & DIRECT-GLce & - & 201.1593 & - & 819 & \\
\hline \multirow[t]{6}{*}{ "8" } & "DIRECT-f" & 19 & -118.700976 & $0.00 \mathrm{E}+00$ & 823 & -118.70 \\
\hline & "UB-DIRECT-f" & 19 & -118.700976 & $0.00 \mathrm{E}+00$ & 797 & \\
\hline & "UB-DIRECT-f+Heur" & 23 & -118.692210 & $0.00 \mathrm{E}+00$ & 689 & \\
\hline & in $[8]$ & 23 & -118.700976 & $0.00 \mathrm{E}+00$ & 881 & \\
\hline & DIRECT-GLc & - & -118.6892 & - & 1197 & \\
\hline & DIRECT-GLce & - & -118.6898 & - & 1947 & \\
\hline
\end{tabular}

\section{Conclusions}

In this paper, we present an extension of the DIRECT method for solving equality and inequality constrained global optimization problems. The extension integrates the filter methodology into the DIRECT and aims to minimize both the objective function and the constraints violation simultaneously. The use of the filter method allows the classification of the hyperrectangles, through the objective and violation values of their center points, in four categories. Features 


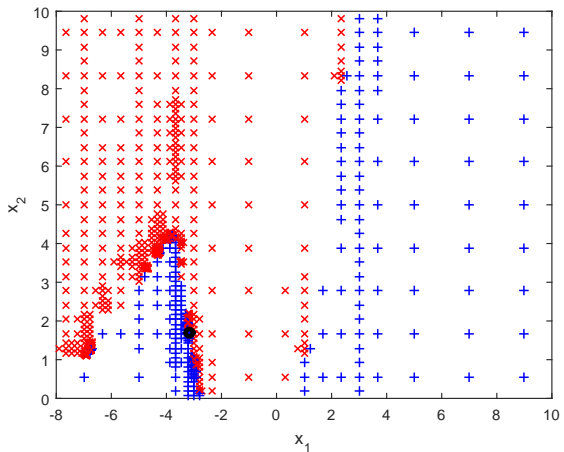

(a) "DIRECT-f" center points

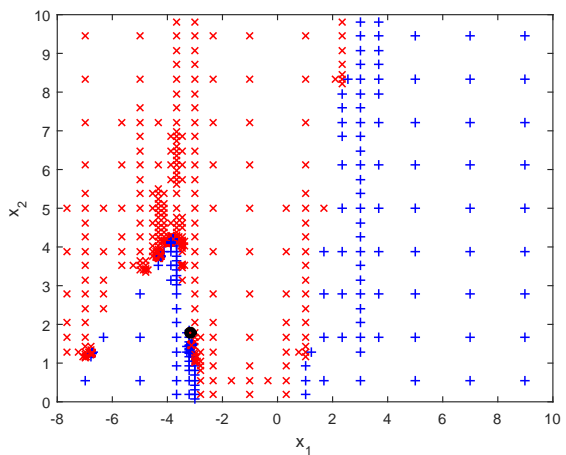

(c) "UB-DIRECT-f+Heur" center points

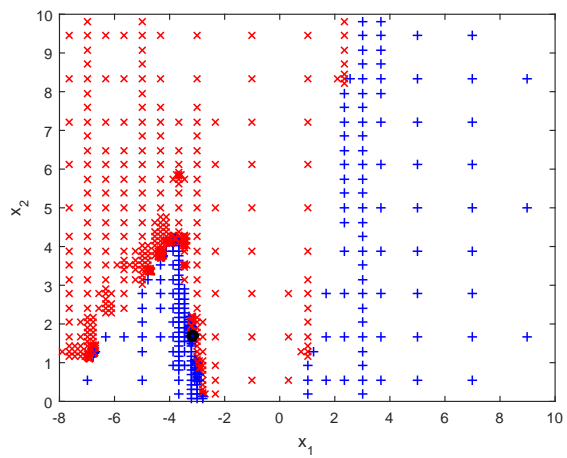

(b) "UB-DIRECT-f" center points

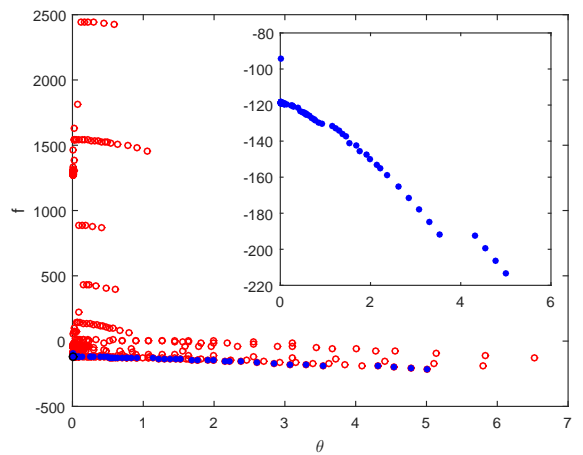

(d) "UB-DIRECT-f+Heur" filter points

Fig. 1. Plots of center points in problem " 8 ": ' + ' (blue) - feasible points and ' $x$ ' (red) infeasible points; 'circle' (red) - dominated points and 'full circle' (blue) - non-dominated points

like non-dominance/dominance and almost feasible/infeasibility are used to classify and separately handle the hyperrectangles. Furthermore, upper bounds on the objective function and on the constraints violation are imposed to identify the hyperrectangles that should be avoided from the process of selecting the most promising hyperrectangles. Furthermore, a heuristic that avoids the identification of potentially optimal hyperrectangles, among those that were mostly divided, has been cyclically (every 10 iterations) implemented.

Preliminary numerical experiments show that the quality and the efficiency of the proposed DIRECT-filter method have been improved when the objective and constraints violation upper bounds are introduced, and in particular, when the heuristic is activated. The comparison carried out with other DIRECT-type methods is encouraging for the smaller dimensional problems. 
Future work will be directed to generate upper bounds based on information gathered from the objective and violation values from each category, resorting to the average and standard deviation of those values. Issues related to the extension of the heuristic to avoid exploring hyperrectangles with the larger sizes, while focusing on hyperrectangles with very small violation and lower objective values, will require further work.

Acknowledgments. The authors wish to thank two anonymous referees for their comments and suggestions to improve the paper. This work has been supported by FCT

- Fundação para a Ciência e Tecnologia within the Projects Scope: UID/CEC/00319/2019 and UID/MAT/00013/2013.

\section{References}

1. Fletcher, R., Leyffer, S.: Nonlinear programming without a penalty function. Mathematical Programming, Series A, 91(2), 239-269 (2002).

2. Jones, D.R., Perttunen, C.D., Stuckman, B.E.: Lipschitzian optimization without the Lipschitz constant. J. Optim. Theory Appl. 79(1), 157-181 (1993)

3. Di Pillo, G., Lucidi, S., Rinaldi, F.: A derivative-free algorithm for constrained global optimization based on exact penalty functions. J. Optim. Theory Appl. 164(3), 862$882(2015)$

4. Stripinis, L., Paulavičius, R., Žilinskas, J.: Penalty functions and two-step selection procedure based DIRECT-type algorithm for constrained global optimization. Struct. Multidisc. Optim. 59(6), 2155-2175 (2019)

5. Jones, D.R.: Direct global optimization algorithm. In: Floudas, C., Pardalos, P. (eds) Encyclopedia of Optimization, pp. 431-440. Springer, Boston MA (2008)

6. Di Pillo, G., Liuzzi, G., Lucidi, S., Piccialli, V., Rinaldi, F.: A DIRECT-type approach for derivative-free constrained global optimization. Comput. Optim. Appl. 65(2), 361-397 (2016)

7. Liu, H., Xu, S., Chen, X., Wang, X., Ma, Q.: Constrained global optimization via a DIRECT-type constraint-handling technique and an adaptive metamodeling strategy. Struct. Multidisc. Optim. 55(1), 155-177 (2017)

8. Costa, M.F.P., Rocha, A.M.A.C., Fernandes, E.M.G.P.: Filter-based DIRECT method for constrained global optimization. J. Glob. Optim. 71(3), 517-536 (2018)

9. Finkel, D.E., Kelley, C.T.: Convergence analysis of the DIRECT algorithm. Technical Report CRSC-TR04-28, Center for Research in Scientific Computation, North Carolina State University (2004)

10. Finkel, D.E., Kelley, C.T.: Additive scaling and the DIRECT algorithm. JJ. Glob. Optim. 36(4), 597-608 (2006)

11. Gablonsky, J.M.: DIRECT version 2.0 user guide. Technical Report CRSC-TR-0108, Center for Research in Scientific Computation, North Carolina State University (2001)

12. Gablonsky, J.M., Kelley, C.T.: A locally-biased form of the DIRECT algorithm. J. Glob. Optim. 21(1), 27-37 (2001)

13. Wu, Y., Ozdamar, L., Kumar, A.: TRIOPT: a triangular-based partitioning algorithm for global optimization. J. Comput. Appl. Math. 177, 35-53 (2005)

14. Sergeyev, Y.D., Kvasov, D.E.: Global search based on efficient diagonal partitions and a set of Lipschitz constants. SIAM J. Optimiz. 16(3), 910-937 (2006) 
15. Liu, Q., Cheng, W.: A modified DIRECT algorithm with bilevel partition. J. Glob. Optim., 60(3), 483-499 (2014)

16. Paulavičius, R., Žilinskas, J.: Simplicial Lipschitz optimization without the Lipschitz constant. J. Glob. Optim., 59(1), 23-40 (2014)

17. Paulavičius, R., Sergeyev Y.D., Kvasov D.E., Žilinskas, J.: Globally-biased DISIMPL algorithm for expensive global optimization. J. Glob. Optim., 59(2-3), 545$567(2014)$

18. Liuzzi, G., Lucidi, S., Piccialli, V.: Exploiting derivative-free local searches in DIRECT-type algorithms for global optimization. Comput. Optim. Appl. 65(2), 449-475 (2016)

19. Sergeyev, Y.D., Kvasov, D.E.: Deterministic Global Optimization: An Introduction to the Diagonal Approach. SpringerBriefs in Optimization Series, Springer-Verlag New York (2017)

20. Stripinis, L., Paulavičius, R., Žilinskas, J.: Improved scheme for selection of potentially optimal hyper-rectangles in DIRECT. Optim. Lett. 12(7), 1699-1712 (2018)

21. Audet, C., Dennis, Jr., J.E.: A pattern search filter method for nonlinear programming without derivatives. SIAM J. Optimiz. 14(4), 980-1010 (2004)

22. Birgin, E.G., Floudas, C.A., Martínez, J.M.: Global minimization using an Augmented Lagrangian method with variable lower-level constraints. Technical Report MCDO121206, January 22, 2007, http://www.ime.usp.br/ egbirgin/ 